Relations industrielles

Industrial Relations

\title{
Index volume 15 - 1960
}

Volume 15, numéro 4, octobre 1960

URI : https://id.erudit.org/iderudit/1021961ar

DOI : https://doi.org/10.7202/1021961ar

Aller au sommaire du numéro

Éditeur(s)

Département des relations industrielles de l’Université Laval

ISSN

0034-379X (imprimé)

1703-8138 (numérique)

Découvrir la revue

Citer ce document

(1960). Index volume 15 - 1960. Relations industrielles / Industrial Relations,

15(4), 527-533. https://doi.org/10.7202/1021961ar

Tous droits réservés (C) Département des relations industrielles de l’Université Laval, 1960
Ce document est protégé par la loi sur le droit d'auteur. L’utilisation des services d'Érudit (y compris la reproduction) est assujettie à sa politique d'utilisation que vous pouvez consulter en ligne.

https://apropos.erudit.org/fr/usagers/politique-dutilisation/ 


\section{NDEX \\ VOLUME 15 - 1960}

Action politique et syndicats, Gérard Filion, (no 4), pp. 496-499.

Anti-cartel, La nouvelle législation, Gérald Marion, (no 4), pp. 425-440.

Assurance-groupe et les conventions collectives, L', Paul Michaud, (no 2), pp. 209224.

Bargaining and Management Rights, Collective, Roger Chartier, (no 3), pp. 298323.

Bien-commun, Action syndicale et, Roger Chartier, (no 4), pp. 483-487.

Cadres, Le syndicalisme des, Jacques Cousineau, s.j., (no 4), pp. 492-496.

Canada, Engineering and Scientific Manpower Resources in, Emile Gosselin, (no 2), pp. 246-248.

Canada, Implications économiques de la protection de la santé au, Claude Morin, ( no 2), pp. 239-246.

Canada, Nouvelle méthode d'estimation du chômage au, Jacques St-Laurent, (no 4), pp. 475-477.

Chômage au Canada, Nouvelle méthode d'estimation du, Jacques St-Laurent, (no 4), pp. 475-477.

Collaboration entre les agents de l'économie, Possibilités et conditions de, JeanRéal Cardin, (no 4), pp. 487-489.

Collective Bargaining and Management Rights, Roger Chartier, (no 3), pp. 298323.

Combines Law, The New, Gérald Marion, (no 4), p. 440.

C.T.C.C. et de la F.T.Q. au gouvernement provincial, Les mémoires de la, JeanRéal Cardin, (no 1), pp. 96-102.

Confessional Unionism and Quebec Labour Law, Gérard Dion (no 2), p. 180.

Confessionnalité syndicale et régime juridique du travail dans le Québec, Gérard Dion, (no 2), pp. 162-179.

Congédier, droit de disposer des hommes, Droit de, Jacques Archambault, (no 4), pp. $410-424$.

C.T.C. au gouvernement fédéral, Mémoire du, (no 2), pp. 269-275.

Contrôle des monopoles, Le, Gérald Marion, (no 1), pp. 118-126.

Conventions collectives, L'Assurance-groupe et les, Paul Michaud, (no 2), pp. 209224. 
Démocratie organique, Syndicats et groupements professionnels dans une, Richard Arès, s.j., (no 4), pp. 480-483.

Démocratisation de l'économie, Syndicalisme et, (no 4), pp. 477-505.

Discipline: a Synthesis Approach, Industrial, Jacques Lucier, (no 3), pp. 359-360.

Discipline industrielle: essai de synthèse, La, Jacques Lucier, (no 3), pp. 350-359

Discipline industrielle, Le système Brown en matière de, Denis Germain, (no 3), pp. 361-371.

Droit de congédier, droit de disposer des hommes, Jacques Archambault, (no 4), pp. $410-424$.

Droit au travail, Le, Gérard Dion, (no 4), pp. 398-409.

Droits de gérance, Négociation collective et, Roger Chartier, (no 3), pp. 323-324.

Economic and Social Consequences of Fringe Benefits, Monteath Douglas, (no 2), pp. 181-192.

Economie, Possibilités et conditions de collaboration entre les agents de $l$, JeanRéal Cardin, ( no 4), pp. 487-489.

Economie, Syndicalisme et démocratisation de l', (no 4), pp. 477-505.

Eglise et l'organisation professionnelle, L', Jean-Guy Hamelin, ptre, (no 4), pp. 499-502.

Employer and Trade Unionism, Government as, Emile Gosselin, (no 2), p. 238.

Engineering and Scientific Manpower Resources in Canada, Emile Gosselin, (no 2) pp. $246-248$.

Familial et revenu familial, Salaire, Gérard Dion, (no 1), pp. 2-30.

Federal Juridiction over Labour Relations: A New Look, F. R. Scott, (no 1), pp. 31-48.

F.T.Q. au gouvernement provincial, Les mémoires de la C.T.C.C. et de la, JeanRéal Cardin, (no 1), pp. 96-102.

Fonction en relations du travail, Pouvoir et, H. D. Woods, (no 4), p. 451.

Fringe Benefits, Economic and Social Consequences of, Monteath Douglas, (no 2), pp. 181-192.

Function in Labour Relations, Power and, H. D. Woods, (no 4), pp. 441-451.

Gérance, Négociation collective et droits de, Roger Chartier, (no 3), pp. 323-324.

Gouvernement-employeur et le syndicalisme, Le, Emile Gosselin, (no 2), pp. 225238.

Gouvernement fédéral, Mémoire du C.T.C. au, (no 2), pp. 269-275.

Gouvernement provincial, Les mémoires de la C.T.C.C. et de la F.T.Q. au, JeanRéal Cardin, (no 1), pp. 96-102.

Government as an Employer and Trade Unionism, Emile Gosselin, (no 2), p. 238. 
Griefs, Les tiers et le règlement des, Roger Chartier, (no 2), pp. 202-208.

Grievances and Third-Party Intervention, Roger Chartier, (no 2), pp. 193-201.

Groupements professionnels dans une démocratie organique, Syndicats et, Richard Arès, s.j., (no 4), pp. 480-483.

Industrial Discipline: a Synthesis Approach, Jacques Lucier, (no 3), pp. 359-360.

Industrial Relations, Sources and References, Jacques Lucier, (no 4), p. 474.

Intervention, Grievances and Third-Party, Roger Chartier, (no 2), pp. 193-201.

Juridiction fédérale dans le domaine du travail, Pour un réaménagement de la, F. R. Scott, (no 1), pp. 49-53.

Juridiction over Labour Relations: A New Look, Federal, F. R. Scott, (no 1), pp. 31-48.

Labor Pluralism at the Local Level, Louis-Marie Tremblay, (no 3), pp. 348-349.

Labour Law, Confessional Unionism and Quebec, Gérard Dion, (no 2), p. 180.

Labour Relations: A New Look, Federal Juridiction over, F. R. Scott, (no 1), pp. 31-48.

Labour Relations, Power and Function in, H. D. Woods, (no 4), pp. 441-451.

Law, The New Combines, Gérald Marion, (no 4), p. 440.

Législation anti-cartel, La nouvelle, Gérald Marion, (no 4), pp. 425-440.

Loi des relations ouvrières, Modification de la, Roger Chartier, (no 1), pp. 102114.

Management Rights, Collective Bargaining and, Roger Chartier, (no 3), pp. 298323.

Manpower Resources in Canada, Engineering and Scientific, Emile Gosselin, (no 2), pp. 246-248.

Mémoire du C.T.C. au gouvernement fédéral, (no 2), pp. 269-275.

Monopoles, Le contrôle des, Gérald Marion, (no 1), pp. 118-126.

Négociation collective et droits de gérance, Roger Chartier, (no 3), pp. 323-324.

New Look, Federal Juridiction over Labour Relations: A, F. R. Scott, (no 1), pp. 31-48.

Organisation professionnelle, L’Eglise et $l$, Jean-Guy Hamelin, ptre, (no 4), pp. 499-502.

Organisation professionnelle: réalisations étrangères, Emile Gosselin, (no 4), pp. 489-492.

Ouvrière au niveau local, Le pluralisme de représentation, Louis-Marie Tremblay, (no 3), pp. 325-348.

Personne humaine, Socialisation et, (no 3), pp. 377-384.

Pluralism at the Local Level, Labor, Louis-Marie Tremblay, (no 3), pp. 348-349. 
Pluralisme de représentation ouvrière au niveau local, Le, Louis-Marie Tremblay, (no 3), pp. 325-348.

Politique et syndicats, Action, Gérard Filion, (no 4), pp. 496-499.

Pouvoir et fonction en relations du travail, H. D. Woods, (no 4), p. 451.

Power and Function in Labour Relations, H. D. Woods, (no 4), pp. 441-451.

Professionnelle: réalisations étrangères, Organisation, Emile Gosselin, (no 4), pp. 489-492.

Propriété dans la société nord-américaine, La, Edward Duff, (no 1), pp. 54-95.

Protection de la santé au Canada, Implications économiques de la, Claude Morin, (no 2), pp. 239-246.

Québec, Confessionnalité syndicale et régime juridique du travail dans le, Gérard Dion, (no 2), pp. 162-179.

Quebec Labour Law, Confessional Unionism and, Gérard Dion, (no 2), p. 180.

Rapport de la Commission Stewart, Le, Roger Charbonneau, (no 1), pp. 115-118.

Régime juridique du travail dans le Québec, Confessionnalité syndicale et, Gérard Dion, (no 2), pp. 162-179.

Reglement des griefs, Les tiers et le, Roger Chartier, (no 2), pp. 202-208.

Relations du travail, Pouvoir et fonction en, H. D. Woods, (no 4), p. 451.

Relations industrielles, La documentation en, Jacques Lucier, (no 4), pp. 452-474.

Relations ouvrières, Modification de la Loi des, Roger Chartier, (no 1), pp. 102-114.

Représentation ouvrière au niveau local, Le pluralisme de, Louis-Marie Tremblay, (no 3), pp. 325-348.

Resources in Canada, Engineering and Scientific Manpower, Emile Gosselin, (no 2), pp. 246-248.

Revenu familial, Salaire familial et, Gérard Dion, (no 1), pp. 2-30.

Salaire familial et revenu familial, Gérard Dion, (no 1), pp. 2-30.

Santé au Canada, Implications économiques de la protection de la, Claude Morin, (no 2), pp. 239-246.

Scientific Manpower Resources in Canada, Engineering and, Emile Gosselin, (no 2), pp. 246-248.

Social Consequences of Fringe Benefits, Economic and, Monteath Douglas, (no 2), pp. 181-192.

Socialisation et personne humaine, (no 3), pp. 377-384.

Société nord-américaine, La propriété dans la, Edward Duff, (no 1), pp. 54-95.

Stewart, Le rapport de la Commission, Roger Charbonneau, (no 1), pp. 115-118.

Syndicale et bien-commun, Action, Roger Chartier, (no 4), pp. 483-487. 
Syndicale et régime juridique du travail dans le Québec, Confessionnalité, Gérard Dion, (no 2), pp. 162-179.

Syndicalisme, Le gouvernement-employeur et le, Emile Gosselin, (no 2), pp. 225237.

Syndicalisme des cadres, Le, Jacques Cousineau, s.j., (no 4), pp. 492-496.

Syndicalisme et démocratisation de l'économie, (no 4), pp. 477-505.

Syndicats, Action politique et, Gérard Filion (no 4), pp. 496-499.

Syndicats et groupements professionnels dans une démocratie organique, Richard Arès, s.j., (no 4), pp. 480-483.

Système Brown en matière de discipline industrielle, Le, Denis Germain, (no 3), pp. 361-371.

Tardini, Lettre du cardinal, (no 4), pp. 477-480.

Trade Unionism, Government as an Employer and, Emile Gosselin, (no 2), p. 238.

Travail, Le droit au, Gérard Dion, (no 4), pp. 398-409.

Travail, Pour un réaménagement de la juridiction fédérale dans le domaine du, F. R. Scott, (no 1), pp. 49-53.

Travail dans le Québec, Confessionnalité syndicale et régime juridique du, Gérard Dion, (no 2), pp 162-179.

Unionism and Quebec Labour Law, Confessional, Gérard Dion, (no 2), p. 180.

\section{JURISPRUDENCE DU TRAVAIL}

Arbitrage en 1958, Mutation et promotion dans les rapports d', (no 2), pp. 263-266.

Arbitrage et certificat de reconnaissance syndicale, Conseil d', (no 2), pp. 266-267. Bref de prohibition et pouvoirs d'enquête de la Commission des relations ouvrières, (no 1), pp. 138-141.

Commission des relations ouvrières, Bref de prohibition et pouvoirs d'enquête de la, ( no 1), pp. 138-141.

Conseil d'arbitrage et certificat de reconnaissance syndicale, (no 2), pp. 266-267.

Contracting Out, Grievance Procedure and Union Liability, (no 2), pp. 249-263.

Convention collective, Griefs après l'expiration de la, (no 3), pp. 372-377.

Convention collective, La procédure de grief à l'expiration de la, (no 1), pp. 126138.

Discharge Case, Areas of Proof in a, (no 4), pp. 508-514.

Dommages, Renonciation aux représailles et recours en, (no 4), pp. 505-508.

Grief à l'expiration de la convention collective, La procédure de, (no 1), pp. 126138.

Griefs après l'expiration de la convention collective, (no 3), pp. 372-377. 
Grievance Procedure and Union Liability, Contracting Out, (no 2), pp. 249-263.

Mutation et promotion dans les rapports d'arbitrage en 1958, (no 2), pp. 263-266.

Procédure de grief à l'expiration de la convention collective, La, (no 1), pp. 126138.

Prohibition et pouvoirs d'enquête de la Commission des relations ouvrières, Bref de, (no 1), pp. 138-141.

Promotion dans les rapports d'arbitrage en 1958, Mutation et, (no 2), pp. 263-266.

Proof in a Discharge Case, Areas of, (no 4), pp. 508-514.

Reconnaissance syndicale, Conseil d'arbitrage et certificat de, (no 2), pp. 266-267.

Renonciation aux représailles et recours en dommages, (no 4), pp. 505-508.

Représailles et recours en dommages, Renonciation aux, (no 4), pp. 505-508.

Rétroactivité dans les rapports d'arbitrage en 1958, La, (no 2), pp. 267-268.

Union Liability, Contracting Out, Grievance Procedure and (no 2), pp. 249-263.

\section{RECENSIONS}

Abegglen, James C., The Japanese Factory, (no 4), pp. 517-518. Bass, Bernard M., Leadership, Psychology and Organizational Behavior, (no 2), p. 281. Beach, L. \& E. L. Clark, Psychology in Business, (no 1), p. 148. Bellows, R., Creative Leadership, (no 1), p. 143. Bennett, W. E., Manager Selection, Education and Training, (no 2), p. 287. Bittel, Leslie R., What Every Supervisor Should Know, (no 2), p. 286. Bolle de Bal, Marcel, Relations humaines et relations industrielles, (no 4), p.519. Bureau international du Travail, Emploi et conditions de travail du personnel infirmier, (no 3), p. 384. Bureau international du Travail, La qualification du travail, (no 3), p. 389. Bureau international du Travail, Protection des fonds et autres bien syndicaux, (no 4), pp. 516-517. Crook, G. Hamilton and Martin Heinstein, The Older Worker in Industry, (no 3), p. 388. Cunningham, W. B., Compulsory Conciliation and Collective Bargaining, (no 1), p. 142. Daloz, Lucien, P. Lethielleux, Le travail selon saint Jean Chrysostome, (no 2), p. 280. Dempsey, Bernard William, The Functional Economy, (no 1), p. 141. Department of Labor, Ottawa, Technological Changes and Skilled Manpower: The Automobile and Parts Manufacturing lndustries, (no 4), pp. 519-520. Direction de l'économique et de la recherche, Ministère du Travail, Ottawa, Organisations ouvrières au Canada, (no 4), p. 514. Drogat Noël, Pays sous-développés et coopération technique, (no 1), p. 145. Dubin, R., Human Relations in Administration, (no 1), p. 142. Ducoin, Georges, Pour une économie du bien commun, (no 3), p. 389. Gray, Robert D., Frontiers of Industrial Relations, (no 1), p. 152. Harbison, Frederick, Management in the Industrial World: An International Analysis, (no 1), p. 146. Harbrecht, Paul, s.j., Pension Funds and Economic Power, (no 1), p. 150. Herzberg, F. \& al, Job Attitudes: Review of Research and Opinion, (no 1), p. 147. Herzberg, F., B. Mausner and B. C. Snyderman, The Motivation to Work, (no 2), p. 275. Hughes, Everett C., Men and Their Work, (no 3), p. 386 . Industrial Relations Centre, McGill University, Montreal, Labour Relations Trends, Retrospect and Prospect, (no 1), p. 151. LeBourre, Raymond, Le syndicalisme français dans la Ve république, (no 2), p. 278. Lebret, L. J., Manifeste pour une civilisation solidaire, (no 3 ), p. 385. Leclerq, Jacques, Du droit naturel à la sociologie, (no 3 ), p. 387. Lehman, G., Physiologie pratique du travail, (no 1), p. 149. Lesieur, Frederick G., The Scanlon Plan, (no 3), p. 387. MacDonald, Lois and Associates, Leadership Dynamics and the Trade-Union Leader, (no 2), p. 283. Merrihue, Willard V., Managing by Communication, (no 2), p. 280. Neuville, Jean, La représentativité 
syndicale, (no 2), p. 289. Pfiffner, John, The Supervision of Personnel, (no 2), p. 277. Rama, Carlos M., Mouvements ouvriers et socialistes, l'Amérique Latine, (no 1), p. 151. Shartel, C. L., Occupational Information, (no 2), p. 276. Slotkin, James Sydney, From Field to Factory: New Industrial Employees, (no 4), pp. 515516. Soule, George H., Longer Life, (no 4), pp. 518-519. Strauss, George and Leonard R. Sayles, Personnel: The Human Problems of Management, (no 3), p. 386. Torrence, George W., Management's Right to Manage, (no 4), pp. 514-515. United States Senate, Readings in Unemployment, (no 2), p. 289. United States Senate, Studies in Unemployment, (no 2), p. 289. Weschler, T. R. \& J. Reisel, Inside a Sensitivity Training Groups, (no 2), p. 285.

Rapport du XVe Congrès des relations industrielles (1960)

\section{DROITS DE GERANCE \\ ET \\ CHANGEMENTS TECHNOLOGIQUES}

Présentation (EMILE Gosselin). Nature et importance des changements technologiques (Jean-Paul Deschênes). Propriété, responsabilité et droits de la gérance (GÉRARD Dion). Efficacité, science, participation à la gestion et droits de gérance (ROGER CHARTIER). Changements technologiques et négociations collectives (JeAn-Réal Cardin). Arbitrabilité des griefs et changements technologiques (JEAN-JACQues GAGNON). Négociabilité et arbitrabilité des changements technologiques (MARIUS BERGERоN). Négociation et arbitrage dans le domaine des changements technologiques (Marcel Pepin, W. Gordon Donnelly, Yvan Legault, Jean SIROIs. Le syndicalisme ouvrier face aux changements technologiques (Lewrs A. Coser).

$$
1 \text { volume - prix } \$ 3.00
$$

\section{LES PRESSES UNIVERSITAIRES LAVAL}

28, rue Ste-Famille,

Québec, P.Q., Canada. 